\title{
SFRP1 Gene
}

National Cancer Institute

\section{Source}

National Cancer Institute. SFRP1 Gene. NCI Thesaurus. Code C95300.

This gene is involved in signaling. 